\title{
PERFIL CINEANTROPOMÉTRICO EM CRIANÇAS DO PROJETO ATLETA DO FUTURO DO SESI DE PRESIDENTE PRUDENTE-SP
}

Leandro Alves da Cunha ${ }^{1,3,4}$, Carlos Augusto de Carvalho Filho ${ }^{1,2,4}$, Ariovaldo de Souza Ribeiro ${ }^{1,2,4}$, Anderson dos Santos Carvalho ${ }^{5}$, Letícia de Mattos Oishi ${ }^{3,4}$, Manoel Carlos Spiguel Lima ${ }^{1,3}$, Elvancir Pereira do Nascimento ${ }^{1}$, José Evaristo Silvério Netto ${ }^{1,2}$

${ }^{1}$ Docente do Curso de Educação Física, ${ }^{2}$ Grupo de Estudos em Atividade Física e Desempenho Esportivo (GEAFiDE), ${ }^{3}$ Grupo de Estudos em Fisiologia do Exercício (GEFE) e ${ }^{4}$ Grupo de Estudos Práticas Pedagógicas em Educação Física Escolar (GEPPEFE') da Universidade do Oeste Paulista - UNOESTE; ${ }^{5}$ Professor efetivo de Educação Física da Autarquia Municipal de Irapuru-SP.

\section{RESUMO}

Os objetivos do presente estudo foram traçar o perfil cineantropométrico e morfológico em escolares (crianças), com idade entre 09 e 10 anos de idade, participantes do Projeto Atleta do Futuro (PAF). Para tanto, foram avaliados 44 crianças, com idades entre 09 e 10 anos. Podemos inferir que as crianças estão dentro do normal em relação à estatura e a massa corporal. Para os testes cineantropométricos as crianças demonstraram ser melhores no teste de 9 minutos de corrida, apresentando bons índices de condicionamento cardiorrespiratório. Já para os testes anaeróbios com teste de velocidade de $20 \mathrm{~m}$ e a corrida de agilidade as crianças avaliadas apresentaram índice abaixo do esperado para suas idades. Demonstraram também, serem mais fortes no teste de abdominal e no arremesso de medicineball se comparados ao teste de salto horizontal. Para o teste de flexibilidade as crianças demonstraram índice abaixo do esperado para suas idades. Estas considerações serão apresentadas a coordenação do PAF, onde serão refletidas e discutidas para o melhor direcionamento das atividades realizadas nas aulas, bem como do projeto em si.

Palavras-chave: cineantropometria, crianças, atleta.

\section{KINANTHROPOMETRY PROFILE IN CHILDREN OF THE FUTURE ATHLETE PROJECT OF THE SESI AT PRESIDENTE PRUDENTE-SP}

\begin{abstract}
The objectives of this study were to profile morphological and kinanthropometric in school (children) aged between 09 and 10 years of age, participants in Project Athlete of the Future (PAF). Thus, we evaluated 44 children, aged between 09 and 10 years. We can infer that children are within normal in height and body mass. For the tests kinanthropometric children were the best in the test run of 9 minutes and showed good levels of cardiorespiratory fitness. As for the tests with anaerobic speed test run of $20 \mathrm{~m}$ and agility of the children assessed had an index lower than expected for their ages. Was also shown to be stronger in the test of abdominal and throwing medicineball compared to the test of horizontal jump. To test the flexibility of the index children showed lower than expected for their ages. These considerations will be presented to coordinate the FAP, which will be reflected and discussed for the better targeting of activities in classrooms, as well as the project itself.
\end{abstract}

Key words: kinanthropometry, children, athlete. 


\section{INTRODUÇÃO}

A palavra Cineantropometria é de origem Grega, sendo que KINES significa "movimento"; ANTHROPO significa "homem", e por fim, METRY "medida", traduzindo podemos entender como "medidas do homem em movimentos", servindo-se para determinação objetiva dos aspectos referentes ao desenvolvimento do corpo humano e das relações existentes entre os componentes físico-motor e a performance (PETROSKI, 2007). Segundo Beuenen e Borms (1990) a ciência Cineantropométrica implica em mensurar e avaliar diversos aspectos do homem em movimento, do nascer ao morrer, bem como, as características físicas do ser humano. Tem ainda o propósito de estudar variações interhumanas, considerando características e qualidades dos indivíduos e/ou de um grupo, bem como, proporciona comparações entre os grupos e intra-grupos.

\section{O Sistema SESI}

O Serviço Social da Indústria é uma entidade de direito privado, nos termos da lei civil, estruturada em base federativa para prestar assistência social aos trabalhadores industriais e de atividades assemelhadas em todo o país. $O$ SESI exerce papel fundamental no desenvolvimento social brasileiro, colaborando efetivamente com a melhoria da qualidade de vida do trabalhador da indústria, seus familiares e comunidade em geral por meio de seus serviços nos campos da educação, saúde, lazer e esporte, cultura, alimentação e outros (SERVIÇO SOCIAL DA INDÚSTRIA, 2006).

\section{O Programa Atleta do Futuro}

O Programa Atleta do Futuro (PAF) do SESI foi criado em 1991 a partir de um plano elaborado conjuntamente com a Universidade de Campinas (UNICAMP). Em 2002 sua metodologia de ensino foi remodelada em parceria com a
Universidade Estadual Paulista (UNESP Campus de Rio Claro). Atualmente é desenvolvido em 44 municípios paulistas atendendo em média mensal de 22.000 alunos na faixa etária entre 7 a 15 anos. A única exigência para ingressar no programa é a comprovação de matrícula escolar.

O PAF viabiliza vagas para filhos de funcionários das indústrias e para a comunidade em geral, o programa conta com o sistema de convênios com prefeituras municipais, associações, empresas beneficiárias e clubes, que geralmente disponibilizam professores para trabalhar no programa.

Embora já tenha passado pelo programa atletas como Danielle Hipólyto e Adrianinha do basquete, entre outros, o intuito não é formar atletas, se estes surgirem, será conseqüência de um trabalho de qualidade. O Objetivo mesmo é que tenhamos alunos cidadãos e futuramente adultos ativos. Dessa forma, o programa também desenvolve durante o ano uma programação paralela com ênfase nos temas transversais e participação das famílias. Assim, por intermédio do esporte, o PAF procura oferecer a oportunidade de inclusão de crianças e jovens em nossa sociedade (SERVIÇO SOCIAL DA INDÚSTRIA, 2008).

\section{OBJETIVOS}

Os objetivos do presente estudo foram traçar o perfil cineantropométrico e da composição corporal em escolares (crianças), com idade entre 09 e 10 anos de idade, participantes do Projeto Atleta do Futuro (PAF) do SESI de Presidente Prudente-SP e referenciar estes dados para classificação orgânica e funcional, quanto à saúde e o desempenho motor dos freqüentadores do referido programa 


\section{METODOLOGIA}

A bateria de avaliação utilizada neste trabalho foi à bateria de testes do Projeto Esporte Brasil (PROESP-BR), projetos lançado pelo Ministério dos Esportes em 2003 visando traçar nacionalmente as características da população infantil e juvenil do país através de avaliações não invasivas utilizadas no âmbito científico da Educação física.

O Proesp-BR objetiva quantificar e qualificar $\mathrm{o}$ organismo em crescimento $\mathrm{e}$ desenvolvimento da população escolar quanto às qualidades morfológicas e a composição corporal, bem como, as capacidades funcionais orgânicas e motoras, após a obtenção dos dados, estes são relacionados com a saúde, com o desempenho motor e com indícios de detecção de talentos esportivos (PROJETO ESPORTE BRASIL, 2004; SILVA, 2005; GAYA, SILVA, 2007).

A consonância entre o PAF e o ProespBR pode ser percebida na compatibilidade de seus objetivos e na perspectiva de subsidiar possíveis intervenções políticas para a área da educação física e esportes em nosso país, que desenvolverá futuramente dois grande eventos desportivos em âmbito mundial a Copa de Futebol em 2014 e as Olimpíadas em 2016.

Foram avaliadas 44 crianças, com idades entre 09 anos ( $n=13$ femininos e $n=09$ masculinos) e 10 anos ( $n=10$ femininos e $n=12$ masculinos), em um único dia, após um período de familiarização. As crianças foram submetidas à aplicação da bateria de avaliação do Proesp-BR, constituída pelas seguintes medidas e testes e na referida seqüência; 1 . Medida de massa corporal; 2. Medida de estatura; 3. Medida de envergadura; 4. Teste "sentar-e-alcançar"; 5. Teste de exercício abdominal; 6. Teste do salto em distância; 7. Teste do arremesso de medicineball; 8. Teste do quadrado; 9. Teste da corrida de 20 metros; 10. Teste dos 9 minutos. Todas as crianças avaliadas pertencem ao PAF do SESI de Presidente Prudente-SP.

\section{RESULTADOS E DISCUSSÃO}

Todos os dados do referido trabalho foram relacionados segundo o "Manual de Aplicação de Medidas e Testes, Normas e Critérios de Avaliação de Conde e Monteiro (2006)". Este Manual apresenta um escore onde os avaliados são classificados como muito fraco, fraco, razoável, bom, muito bom e excelente.

Para dados antropométricos (Tabela 1) todos os grupos encontra-se com o IMC dentro da faixa de normalidade. Porém, vale lembrar que o IMC apresenta limitação para classificar crianças com menos de 10 anos de idade.

Tabela 1. Distribuição da amostra estratificada pela idade e por sexo com representação do $n$, da média e do desvio padrão para as medidas de estatura, da massa corporal, índice de massa corporal (IMC) e envergadura, em escolares participantes do PAF do SESI de Presidente Prudente-SP.

\begin{tabular}{ccccccc}
\hline Idade & Sexo & $\mathbf{n}$ & $\begin{array}{c}\text { Estatura } \\
(\mathrm{m})\end{array}$ & $\begin{array}{c}\text { Massa Corporal } \\
(\text { Peso Kg })\end{array}$ & $\begin{array}{c}\text { IMC } \\
\left(\mathrm{Kg} / \mathrm{m}^{2}\right)\end{array}$ & $\begin{array}{c}\text { Envergadura } \\
(\mathrm{cm})\end{array}$ \\
\hline 9 & $\mathrm{M}$ & 09 & $1,33 \pm 0,10$ & $30,82 \pm 6,99$ & $17,17 \pm 1,90$ & $1,34 \pm 0,12$ \\
9 & $\mathrm{~F}$ & 13 & $1,33 \pm 0,04$ & $28,87 \pm 3,41$ & $16,18 \pm 1,24$ & $1,35 \pm 0,05$ \\
10 & $\mathrm{M}$ & 12 & $1,36 \pm 0,04$ & $31,44 \pm 4,13$ & $16,86 \pm 1,30$ & $1,40 \pm 0,05$ \\
10 & $\mathrm{~F}$ & 10 & $1,39 \pm 0,06$ & $34,01 \pm 6,50$ & $17,48 \pm 2,74$ & $1,42 \pm 0,07$ \\
\hline
\end{tabular}


Tabela 2. Distribuição da amostra estratificada pela idade e por sexo com representação do $n$, da média e do desvio padrão para o teste de corrida de 9 minutos, teste abdominal em 1 minuto e teste de flexibilidade linear em escolares participantes do PAF do SESI de Presidente Prudente-SP.

\begin{tabular}{cccccc}
\hline Idade & Sexo & $\mathbf{n}$ & $\begin{array}{c}\text { Corrida de 9 minutos } \\
(\mathrm{m})\end{array}$ & $\begin{array}{c}\text { Abdominal } \\
\text { (Repetições em 1') }\end{array}$ & $\begin{array}{c}\text { Flexibilidade Linear } \\
\text { Sentar-e-alcançar }(\mathrm{m})\end{array}$ \\
\hline 9 & $\mathrm{M}$ & 09 & $1471,25 \pm 218,73$ & $35,44 \pm 5,34$ & $26,06 \pm 4,03$ \\
9 & $\mathrm{~F}$ & 13 & $1222,73 \pm 235,67$ & $30,98 \pm 7,59$ & $25,00 \pm 4,98$ \\
10 & $\mathrm{M}$ & 12 & $1462,00 \pm 214,48$ & $37,33 \pm 9,89$ & $24,83 \pm 6,62$ \\
10 & $\mathrm{~F}$ & 10 & $1254,17 \pm 226,02$ & $30,20 \pm 6,00$ & $22,80 \pm 5,90$ \\
\hline
\end{tabular}

Para dados cineantropométricos (Tabela 2) o grupo com 09 anos masculino apresentou-se com índice muito bom e os demais grupos com índice bom para a corrida de 9 minutos. Para o teste de abdominal em 1', o grupo de 10 anos feminino apresentou-se com índice bom e os demais grupos com índice muito bom. Para o teste de flexibilidade o grupo de 09 anos masculino o apresentou-se com índice fraco e os demais grupos com índice muito fraco.

Para dados cineantropométricos (Tabela 3) o grupo com 09 anos masculino e o grupo com 10 anos feminino apresentou-se com índice fraco

Tabela 3. Distribuição da amostra estratificada pela idade e por sexo com representação do $n$, da média e do desvio padrão para o teste do quadrado, teste de salto horizontal, teste de arremesso de medicineball e teste de corrida em velocidade de 20 em escolares participantes do PAF do SESI de Presidente Prudente-SP.

\begin{tabular}{ccccccc}
\hline Idade & Sexo & $\mathbf{n}$ & $\begin{array}{c}\text { Agilidade } \\
\text { Quadrado } \\
(\mathrm{s})\end{array}$ & $\begin{array}{c}\text { Salto } \\
\text { Horizontal } \\
(\mathrm{cm})\end{array}$ & $\begin{array}{c}\text { Arremesso } \\
\text { Medicineball } \\
(\mathrm{cm})\end{array}$ & $\begin{array}{c}\text { Velocidade } \\
\mathbf{2 0} \text { metros } \\
(\mathrm{s})\end{array}$ \\
\hline 9 & $\mathrm{M}$ & 09 & $7,40 \pm 0,71$ & $133,00 \pm 11,90$ & $240,22 \pm 48,71$ & $4,88 \pm 0,17$ \\
9 & $\mathrm{~F}$ & 13 & $7,67 \pm 0,51$ & $122,25 \pm 12,61$ & $216,29 \pm 30,24$ & $5,03 \pm 0,34$ \\
10 & M & 12 & $6,96 \pm 0,66$ & $133,92 \pm 19,27$ & $247,08 \pm 36,40$ & $4,62 \pm 0,28$ \\
10 & F & 10 & $7,57 \pm 0,44$ & $117,60 \pm 6,20$ & $230,00 \pm 30,64$ & $5,03 \pm 0,31$ \\
\hline
\end{tabular}

\section{CONCLUSÃO}

Podemos inferir que os resultados encontrados demonstram que as crianças estão dentro do esperado em relação à estatura e a massa corporal. Para os testes e os grupo com 09 anos feminino e com 10 anos masculinos apresentou índice razoável para o teste de agilidade. Para o teste de salto vertical, o grupo com 09 anos masculino e feminino apresentou índice razoável e os grupos com 10 anos masculinos e femininos apresentou índice fraco. Para o teste de arremesso de medicineball todos os grupos apresentaram índice bom. Por fim, para o teste de velocidade de $20 \mathrm{~m}$, todos os grupos apresentaram índice muito fraco. 
de velocidade de corrida de $20 \mathrm{~m}$ e a corrida de agilidade. Demonstraram também, serem mais fortes no teste de abdominal e no arremesso de medicineball se comparados ao teste de salto horizontal. Para 0 teste de flexibilidade as crianças demonstraram índice abaixo do esperado para suas idades

A realização desta pesquisa contempla o PAF alicerçando o planejamento do programa classificando os organismos das crianças freqüentadores, a fim de detectar déficit de crescimento, sobrepeso e/ou obesidade infantil para que o responsável legal seja alertado e informado sobre as cabíveis intervenções minimizadoras destas nuances, bem como, orientar os responsáveis pelas atividades desportivas e recreativas para as potencialidade e fragilidades na aptidão física destas crianças.

\section{REFERÊNCIAS}

Beunen G, Borms J. Kinanthropometry: roots, developments and future. Journal of Sports Sciences 1990; 8:1-15. http://dx.doi.org/10.1080/02640419008732127

Gaya A, Silva G. Projeto Esporte Brasil: observatório permanente dos indicadores de saúde e fatores de prestação esportiva em crianças e jovens. Manual de aplicação de medidas e testes, normas e critérios de avaliação. [Porto Alegre]: Universidade Federal do Rio Grande do Sul, Centro de Excelência Esportiva; 2007. [acesso em 2010 jul 21]. Disponível em: http://blog.educacional.com.br/susana_paraiba/file s/proesp-2007.pdf

Petroski E L. Antropometria: técnicas e padronização. 3.ed. Blumenau: Nova Letra; 2007.

Projeto Esporte Brasil. Indicadores de saúde e fatores de prestação esportiva em crianças e jovens Manual de aplicação de medidas e testes somatomotores protocolo da Rede Cenesp. [Porto Alegre]: CENESP-UFRGS; 2004.

Serviço Social da Indústria. Programa Atleta do Futuro (PAF); 2006. [acesso em 2010 jul 11]. Disponível em: http://www.sesisp.org.br/home/2006/institucional/ Serviço Social da Indústria. Programa Atleta do Futuro (PAF); 2008. [acesso em 2010 jul 11]. Disponível em: http://www.fiesp.com.br/agencianoticias/2008/07/0 8/sesi_olimpaf.pdf.

Silva G M G. Talento esportivo: um estudo dos indicadores somatomotores na seleção de jovens escolares [dissertação]. Porto Alegre: Universidade Federal do Rio Grande do Sul; 2005. 